\title{
Expected Qualifications for External Coaches in School-Based Extracurricular Sports Activities
}

\section{Dr. Kenryu Aoyagi (Corresponding Author)}

Faculty of Sport Sciences, Waseda University, 2-579-15 Mikajima, Tokorozawa, Saitama 359-1192, Japan Japan Society for the Promotion of Science, 5-3-1 Koujimachi, Chiyoda, Tokyo 102-0083, Japan

Email:kenryu.aoyagi@gmail.com

\section{Dr. Kaori Ishii}

Faculty of Sport Sciences, Waseda University, 2-579-15 Mikajima, Tokorozawa Saitama 359-1192, Japan Email: ishiikaori@aoni.waseda.jp

\section{Dr. Ai Shibata}

Faculty of Health and Sport Sciences, University of Tsukuba, 1-1-1 Tennodai, Tsukuba Ibaraki 305-8574, Japan Email: shibata@taiiku.tsukuba.ac.jp

\section{Dr. Hirokazu Arai}

Faculty of Letters, Hosei University, 2-17-1 Fujimi, Chiyoda, Tokyo 102-8160, Japan Email: ICB53570@nifty.com

\section{Dr. Koichiro Oka}

Faculty of Sport Sciences, Waseda University, 2-579-15 Mikajima, Tokorozawa Saitama 359-1192, Japan Email: koka@waseda.jp

\section{Doi:10.5901/jesr.2015.v5n3p53}

\section{Abstract}

This study aimed to examine the importance of expected qualifications for external coaches and their differences between junior high and high schools. The participants were 253 teachers at public junior high and public high schools. A crosssectional self-administrated questionnaire survey was conducted. The questionnaire was developed based on a previous interview study among teachers. Percentages of applicable response were calculated to evaluate the requirements for each qualification. Chi-square tests and Fisher's exact test were used for analysis. As results, "humanity," "ability," "cooperativeness," and "trust" were highly expected. Conversely, expertise in areas other than technical coaching, age, and occupation were not highly perceived as expected qualifications. Further, there were no considerable differences between junior high and high schools. It is necessary to develop guidelines for recruiting external coaches that reflect the highly expected qualifications found in this study. It would also be useful to conduct workshops to convey strongly expected qualifications to external coaches. However, expertise for areas other than technical coaching, age, occupation, and differences between junior high and high schools have low priority in the recruitment strategy for external coaches. This study adds a quantitative evaluation to the expected qualifications identified in a previous report. Investigating these expected qualifications using a quantitative method would be valuable toward improving the recruitment system for external coaches.

Keywords: human resource management; human resource development; extracurricular activity; physical education; volunteer

\section{Introduction}

Large numbers of children and adolescents participate in school-based extracurricular sports activities (SBECSA) after school or on weekends and engage in sports and physical activities (Australian Bureau of Statistics, 2012; Edwards, Kanters, \& Bocarro, 2011; Ministry of Education, Culture, Sports, Science and Technology in Japan [MEXT], 2013; Sport Council Wales, 2009). These SBECSA have many positive benefits for young people's physical, mental, academic, and 
social development (Barnett, 2007; Dotterer, McHale, \& Crouter, 2007; Fredricks \& Eccles, 2006; Lipscomb, 2007; MEXT, 2012; Schaefer, Simpkins, Vest, \& Price, 2011; Shernoff \& Vandell, 2007). Promotion of youth participation in SBECSA is thus important.

The need for external coaches in SBECSA has increased over recent years (Macdonald, 2011; MEXT, 2013; Wiersma \& Sherman, 2005; Williams, Hay, \& Macdonald, 2011). This is due to the lack of in-school coaches (i.e. teachers) who are able to coach SBECSA expertly, the aging of teachers (Akita Prefecture Board of Education, 2013), and excessive workload burden for SBECSA teachers (Japan Senior High School Teachers Union, 2008; MEXT, 1997; Whiteley \& Richard, 2012). External coaches are therefore considered a vital human resource in addressing these problems and maintaining active SBECSA.

However, extortion and violence have been reported as problems caused by external coaches (Shioya, 2002; Tokyo Metropolitan Board of Education, 2008; Wiersma \& Sherman, 2005). Further, external coaches may not be beneficial for youth education since they sometimes focus excessively on skill development and winning (Aoyagi et al., 2013; Flintoff, 2008). Accordingly, recruiting appropriate external coaches for SBECSA is an important issue. Aoyagi et al. (2013) conducted semi-structured interviews for teachers to identify qualitatively categories of expected qualifications for external coaches; the results included "humanity," "ability," "cooperativeness," "attributions," and "trust." However, that study addressed only the classifications of expected qualifications-not the extent of teachers' expectations for each qualification. Assessing those qualifications extracted by qualitative research from a quantitative method-called mixedmethods approach (Creswell, 2014)-is important to develop effective recruitment strategies that large population of teachers could adopt. Because there are not even descriptive data, the present study primary aimed to examine the percentages of teachers who expect external coaches to have each qualification identified in the previous qualitative study (Aoyagi et al., 2013).

In this regard, it is also necessary to examine differences among the various types of schools owing to potential differences in their coaching needs (Flintoff, Foster, \& Wystawnoha, 2011). In Japan, it is appropriate to focus on the differences between junior high and high schools since most SBECSA are performed in such schools. The other purpose of the present study was therefore describing the differences between junior high and high schools.

\section{Methods}

\subsection{Participants and Procedures}

The participants in the present study were teachers who worked at public junior high or public high schools. A crosssectional self-administrated questionnaire survey was sent to them in 2012. Request letters for participation in the study along with a set of questionnaires (including instructions and a consent form) were delivered to the principals of 188 randomly selected schools (94 junior high and 94 high schools). All 47 prefectures in Japan were represented. Two junior high and two high schools were selected from each prefecture. Unified junior high and high schools, evening schools, and branch schools were excluded before the random sampling because they are a minority in the school system and may have biased the results. To avoid sampling bias, such as only physical education teachers answering the questionnaire, 10 sets of questionnaires were sent to each school. Therefore, the maximum numbers of participants was 1,880. The school principal distributed the questionnaire to the teachers. Then, completed surveys were returned to the researchers. All participants were informed of the purpose and design of the study before provided written informed consent. The research proposal was approved by the university ethics board.

\subsection{Questionnaire}

The questionnaire contained 52 items for assessing the expected qualifications for external coaches in SBECSA. The items covered all expected qualifications found in the previous qualitative study (Aoyagi et al., 2013). This was the basic question: "If you used an external coach, which of the following qualifications would you look for in such a coach." Responses were set on a six-point scale ranging from "not applicable at all (0)" to "very applicable (5)." Each participant was also asked to answer a series of sociodemographic questions about their gender, age, type of school (junior high or high school), teaching subject, and whether or not they undertook any SBECSA and made use of external coaches. 


\subsection{Analyses}

Percentages of applicability were calculated to evaluate the requirement for each qualification. Responses of $0-2$ were defined as inapplicable answers, and 3-5 were considered applicable. Percentages of applicability among SBECSA teachers who worked in junior high and high schools were calculated separately. Chi-square tests $(\alpha=0.05)$ were conducted to verify differences between the two groups. Fisher's exact test was used if the chi-square test was not appropriate for analysis. Any missing values were excluded. All analyses were conducted using IBM SPSS Statistics Version 21.

\section{Results}

\subsection{Participant Characteristics}

Responses were received from 253 teachers (response rate, 13.5\%). Their characteristics appear in Table 1. There were 178 males (70.4\%) and 74 females (29.2\%). The participants had a wide age range, with a mean age of 42.0 years (standard deviation, 9.8). The numbers of teachers who worked at junior high and high schools were $107(42.3 \%)$ and $142(56.1 \%)$, respectively. There were 71 physical education teachers (28.1\%); 228 teachers (90.1\%) undertook SBECSA, and 65 SBECSA teachers (25.7\%) used an external coach.

Table 1. Characteristics of participants

\begin{tabular}{lcc}
\hline & $\mathrm{n}$ & $\%$ \\
\hline Overall & 253 & 100.0 \\
Gender & & \\
Male & 178 & 70.4 \\
Female & 74 & 29.2 \\
Missing & 1 & 0.4 \\
Age group & & \\
23-29 & 33 & 13.0 \\
30-39 & 70 & 27.7 \\
40-49 & 81 & 32.0 \\
50-60 & 68 & 26.9 \\
Missing & 1 & 0.4 \\
Type of school & & \\
Junior high & 107 & 42.3 \\
High & 142 & 56.1 \\
Missing & 4 & 1.6 \\
Teaching subject & \multicolumn{3}{c}{} \\
Physical education and Health & 71 & 28.1 \\
Others & 178 & 70.4 \\
Missing & 4 & 1.6 \\
Status of SBECSA & \multicolumn{3}{c}{} \\
Engaged and use external coach & 65 & 25.7 \\
Engaged but don't use external coach & 163 & 64.4 \\
Not engaged & 25 & 9.9 \\
Missing & 0 & 0.0 \\
\hline
\end{tabular}

\subsection{Expected Qualifications for External Coaches}

The percentages of the applicable responses were calculated for all participants and with respect to each school type (Table 2). In terms of "humanity," all the items in "abidance by rules" were indicated by over $90 \%$ of the participants. The other items in the "humanity" category_-"character," "educational thinking," and "no business use"-were selected by over $80 \%$ of the participants. In terms of "ability," although the two items in the subcategory of "technical coaching" were chosen by $90 \%$ or more of the participants, the other expected qualifications were low except for the following: no. 26. "having credentials for coaching" (70.3\%) and no. 30 "having experience in coaching" (76.2\%). Expertise in areas other 
than technical coaching was much less of a requirement.

In the category of "cooperativeness," all items in the "communication skill" subcategory were selected by over $90 \%$ of the participants. High percentages were also achieved for the following in the "support of SBECSA teachers" subcategory: no. 36 "being an adjunct of SBECSA teacher" (82.3\%); no. 37 "coaching regularly" (97.6\%); and no. 39 "giving main position to SBECSA teacher" (77.8\%). Expectations for the "age" and "occupation" subcategories of the "attributions" category were only $30 \%$ or less. With regard to the "trust" category, only no. 49 "understanding the character of the person" (96.8\%) achieved a high percentage. Other items in that category were not highly rated, such as no. 47 "pupil the teacher once taught" (9.2\%) and no. 51 "parent of the team members" (8.8\%).

The items that showed significantly higher proportions among junior high school teachers were as follows: no. 36 "being an adjunct of SBECSA teacher" (89.5\% versus $76.6 \%$ for high school teachers; chi-square $=6.849, p=0.009$ ) and no. 51 "parent of the team members" (13.3\% versus 5.7\%; chi-square $=4.336, p=0.037$ ). By contrast, with four items, there were significantly higher proportions among high school teachers: no. 9 "no physical punishment" (97.9\% versus $90.3 \%$ for junior high school teachers; chi-square $=6.782, p=0.009$ ); no. 12 "having clear boundaries with team members" (99.3\% versus 94.3\%; by Fisher's exact test, $p=0.044)$; no. 16 "not only engaging in technical coaching" (93.6\% versus 83.8\%; chi-square $=6.027, p=0.014)$; and no. 47 "pupil the teacher once taught" $(12.1 \%$ versus $4.8 \%$; chi-square $=3.933, p=0.047$ ).

Table 2. Percentages for expected qualifications of external coaches by teachers

\begin{tabular}{|c|c|c|c|c|c|}
\hline \multirow{2}{*}{ Large category (5) } & \multirow{2}{*}{ Middle category (14) } & \multirow{2}{*}{ Small category (52) } & \multirow{2}{*}{ Overall (\%) } & \multicolumn{2}{|c|}{ School type } \\
\hline & & & & Junior high & High \\
\hline \multirow{18}{*}{ humanity } & \multirow[t]{8}{*}{ character } & 1. a person of integrity & 95.2 & 96.2 & 95.0 \\
\hline & & 2. being trusted by team members & 98.0 & 98.1 & 97.9 \\
\hline & & 3. having passion & 99.2 & 98.1 & 100.0 \\
\hline & & 4. having general intelligence & 99.2 & 99.0 & 99.3 \\
\hline & & 5. a person who likes children & 92.8 & 92.4 & 92.9 \\
\hline & & 6. cheerful disposition & 86.7 & 89.5 & 84.4 \\
\hline & & 7. a person who does not hide anything & 81.5 & 82.9 & 80.9 \\
\hline & & 8. a person who likes sports & 80.3 & 76.2 & 83.7 \\
\hline & \multirow[t]{5}{*}{ abidance by rules } & 9. no physical punishment & 94.7 & 90.3 & $97.9^{* \star}$ \\
\hline & & 10. staying on time & 97.2 & 96.2 & 97.9 \\
\hline & & 11. no sexual harassment & 98.0 & 97.1 & 98.6 \\
\hline & & 12. having clear boundaries with team members & 97.2 & 94.3 & $99.3^{*}$ \\
\hline & & 13. abiding by a duty of secrecy & 98.0 & 98.1 & 97.9 \\
\hline & \multirow[t]{4}{*}{ educational thinking } & 14. thinking of the personal progress of team members & 98.4 & 97.1 & 99.3 \\
\hline & & 15. understanding that SBECSA is a school activity & 95.6 & 95.2 & 95.7 \\
\hline & & 16. not only engaging in technical coaching & 89.5 & 83.8 & $93.6^{*}$ \\
\hline & & 17. not only valuing winning & 88.0 & 90.5 & 85.8 \\
\hline & no business use & 18. not using status of external coach in other business & 84.7 & 87.6 & 82.3 \\
\hline \multirow{13}{*}{ ability } & \multirow[t]{6}{*}{ expert } & 19. expert in physical training & 50.2 & 49.5 & 51.1 \\
\hline & & $\begin{array}{l}\text { 20. expert with a high level technique and coach in the } \\
\text { short-term }\end{array}$ & 55.4 & 58.1 & 54.6 \\
\hline & & 21. ability to perform acupuncture or massage & 18.7 & 14.4 & 21.6 \\
\hline & & 22. expert in mental training & 42.3 & 42.3 & 42.6 \\
\hline & & 23. a person who can engage more than one SBECSA & 14.9 & 12.5 & 16.3 \\
\hline & & 24. expert in nutritional guidance & 43.0 & 37.1 & 47.5 \\
\hline & \multirow[t]{3}{*}{ credentials } & 25. taken a course in coaching & 61.0 & 59.0 & 63.1 \\
\hline & & 26. having credentials for coaching & 70.3 & 70.5 & 70.2 \\
\hline & & 27. having teaching credentials & 14.5 & 12.4 & 16.3 \\
\hline & \multirow[t]{2}{*}{ technical coaching } & 28. being able to coach technically & 98.8 & 98.1 & 99.3 \\
\hline & & 29. having knowledge about technical coaching theory & 90.3 & 91.3 & 89.3 \\
\hline & \multirow[t]{2}{*}{ experience } & 30. having experience in coaching & 76.2 & 71.2 & 79.4 \\
\hline & & 31. having experience in teaching & 14.9 & 14.3 & 14.9 \\
\hline \multirow{8}{*}{ cooperativeness } & \multirow[t]{4}{*}{ communication skill } & 32. being able to communicate with others & 98.0 & 98.1 & 97.9 \\
\hline & & 33. fit of coaching policy with SBECSA teacher & 95.6 & 96.2 & 95.0 \\
\hline & & 34. not coaching only by the external coach's opinion & 94.4 & 95.2 & 93.6 \\
\hline & & 35. ability to communicate opinions to SBECSA teacher & 92.0 & 95.2 & 89.4 \\
\hline & \multirow[t]{4}{*}{ support of SBECSA teachers } & 36. being an adjunct of SBECSA teacher & 82.3 & $89.5^{\star \star}$ & 76.6 \\
\hline & & 37. coaching regularly & 97.6 & 97.1 & 97.9 \\
\hline & & $\begin{array}{l}\text { 38. becoming a bridge between SBECSA teacher and } \\
\text { team members }\end{array}$ & 67.9 & 70.5 & 66.0 \\
\hline & & 39. giving main position to SBECSA teacher & 77.8 & 81.9 & 74.3 \\
\hline
\end{tabular}




\begin{tabular}{|c|c|c|c|c|c|}
\hline \multirow{7}{*}{ attributions } & age & 40. young & 20.8 & 18.6 & 22.1 \\
\hline & & 41. age from 30 s to $40 \mathrm{~s}$ & 16.1 & 15.2 & 16.4 \\
\hline & & 42. elderly & 4.8 & 4.8 & 5.0 \\
\hline & & 43. younger than SBECSA teacher & 11.2 & 9.5 & 12.8 \\
\hline & occupation & 44. civil servant & 5.2 & 3.8 & 6.4 \\
\hline & & 45. a person whose job is coaching SBECSA & 30.0 & 25.0 & 32.1 \\
\hline & & 46. sport store staff & 4.0 & 4.8 & 3.5 \\
\hline \multirow{6}{*}{ trust } & acquaintances & 47. pupil the teacher once taught & 9.2 & 4.8 & $12.1^{*}$ \\
\hline & & 48. introduction from acquaintance & 27.7 & 21.9 & 31.9 \\
\hline & & 49. understanding the character of the person & 96.8 & 96.2 & 97.2 \\
\hline & & 50. graduate student of the school & 10.8 & 10.5 & 9.9 \\
\hline & & 51. parent of the team members & 8.8 & $13.3^{*}$ & 5.7 \\
\hline & selection by SBECSA teacher & 52. selection by SBECSA teacher & 63.0 & 62.1 & 63.6 \\
\hline
\end{tabular}

Note. ${ }^{* *}=p<0.01 ;{ }^{*}=p<0.05$; Percentages exclude missing data.

\section{Discussion}

In the present study, a cross-sectional self-administrated questionnaire survey was conducted among teachers to examine the importance of the expected qualifications for external coaches. The differences in perceptions between teachers in junior high and high schools were also evaluated. The mainly reported expected qualifications of external coaches by teachers were as follows: "humanity" (e.g., appropriate "character," "abidance by rules," and "educational thinking"), "ability" (especially having coaching credentials and experience, and technical coaching skills and knowledge), "cooperativeness" ("communication skill" and "support of SBECSA teachers"), and "trust."

Recently, there has been growing concern about physical punishment in Japanese schools. Accordingly, MEXT (2013) has produced a guideline toward preventing such punishment. With this context, many participants in the present study may have been particularly conscious of the need to abide by rules.

Flintoff et al. (2011) stressed the importance of coaching with educational thinking, especially in elementary schools. The results of the present study indicate that educational thinking is also a strong requirement for external coaches in junior high and high schools. Therefore, educational thinking could be considered an essential qualification for external coaches in all school stages.

Cooperativeness was also highly perceived in the present study. Aoyagi et al. (2013) found that teachers' concerns about the ill-defined status of external coaches and teachers was a barrier to their recruitment: an external coach having stronger influence on students than an SBECSA teacher could cause problems in teacher-student relations. Thus, cooperativeness on the part of the external coaches in maintaining the dominant position of teachers is an important factor in recruiting coaches.

The present study found that over $70 \%$ of teachers required that external coaches have coaching credentials. Aoyagi, Ishii, Shibata, Arai, and Oka (2014) reported that individuals with coaching credentials were about eight times more likely to be motivated to become external coaches than coaches without such credentials. Coaches with the appropriate credentials would therefore appear to be an appropriate potential human resource. Japanese local governments (e.g., boards of education) and mediating organizations (e.g., sports leader bank: Sasakawa Sports Foundation, 2014) should obtain information about the needs of external coaches from schools and convey such information to coaches with credentials. However, one study found that the unknown character of the registered individuals was an impediment to teachers' use of such a mediation system (Kanagawa Prefectural Center of Physical Education, 2007). The present study found that being able to understand the character of the coach was more important than their relationship with the teachers (or students or parents of students). Hence, with a mediation system, information about the character of registrants is essential for the system to be effective. If disclosing information about the character of registrants on a Web site is difficult because of privacy protection, it is recommended that local governments operate a mediating system or work with mediating organizations to conduct interviews and check the registrants' character before a formal decision is made about their registration. It is also necessary to allow teachers to conduct interviews before formal recruitment of external coaches takes place.

This study found that expertise in areas other than technical coaching, age, and occupation were not accorded high importance. In addition, there were no considerable differences between junior high and high schools. Item no. 36 "being an adjunct of SBECSA teacher" was highly perceived in junior high schools; no. 9 "no physical punishment," no. 12 "having clear boundaries with team members," and no. 16 "not only engaging in technical coaching" were statistically high in high schools. However, the response to these items in both school types indicated their high value. Thus, qualifications for expertise in areas other than technical coaching, age, occupation, and the differences between junior 
high and high schools have low priority with respect to recruitment strategy for external coaches.

The present study has some limitations. First, although the participants were collected nationwide, the representativeness of the participants may have been insufficient owing to the low response rate. Therefore, careful discussion is required regarding the generalization of the results of this study. Second, this study used a selfadministrated questionnaire; however, the questionnaire content was sound, having been developed through careful interviews based on previous findings (Aoyagi et al., 2013). Despite these limitations, the present study adds quantitative evaluation to the expected qualifications reported by Aoyagi et al. (2013). Using these expected qualifications based on a quantitative method-the mixed-methods approach adopted by Creswell (2014)—would be valuable toward improving the recruitment system for external coaches. Future studies should evaluate how many external coaches have the expected qualifications or how those qualifications of external coaches may be improved by workshop interventions.

\section{Conclusion}

The results from the cross-sectional self-administrated questionnaire survey given to teachers indicated that "humanity," "ability," "cooperativeness," and "trust" were highly expected. By contrast, expertise for areas other than technical coaching, age, and occupation were not highly perceived as expected qualifications. There were no considerable differences between junior high and high schools. This study has practical implications for local governments that operate a mediating system or work with mediating organizations. First, it is necessary to develop guidelines for the recruitment of external coaches that reflect the results of the present study. Second, it would be useful to set up workshops to convey the highly expected qualifications to external coaches and promote participation in them by coaches. Third, it is essential to provide information about the character of external coaches to teachers. It is also necessary to determine the needs for external coaches of schools and convey these needs to coaching credential holders. Fourth, it is recommended that mediating organizations conduct interviews among registrants and that their character be checked before formal registration. There is also a need for teachers to be encouraged to conduct candidate interviews before formal recruitment of external coaches takes place. If a local community lacks such a mediating system, establishing this type of system could promote the recruitment of appropriate external coaches.

\section{Acknowledgements}

This study was supported by Grant No. 26-3450 from the Japan Society for the Promotion of Science and the MEXTSupported Program for the Strategic Research Foundation at Private Universities, 2015-2020 from the Ministry of Education, Culture, Sports, Science and Technology (MEXT).

\section{References}

Akita Prefecture Board of Education. (2013). The way of school-based extracurricular sports activity in school education (Improved Edition). Retrieved from http://www.pref.akita.Ig.jp/www/contents/1381813119788/files/tebiki4.pdf (January 22, 2015)

Aoyagi, K., Ishii, K., Shibata, A., Arai, H., Hibi, C., \& Oka, K. (2013). Factors associated with teachers' recruitment and continuous engagement of external coaches in school-based extracurricular sports activities: A qualitative study. Advances in Physical Education, 3(2), 62-70. doi: 10.4236/ape.2013.32010

Aoyagi, K., Ishii, K., Shibata, A., Arai, H., \& Oka, K. (2014). Sociodemographic characteristics of potential external coaches for schoolbased extracurricular sports activities. Journal of Japan Society of Sports Industry, 24(2), 185-193. doi: 10.5997/sposun.24. 2_185

Australian Bureau of Statistics. (2012). Children's participation in cultural and leisure activities, Australia, Apr 2012. Retrieved from http://www.abs.gov.au/ausstats/abs@.nsf/mf/4901.0 (January 22, 2015)

Barnett, L. A. (2007). 'Winners' and 'losers': The effects of being allowed or denied entry into competitive extracurricular activities. Journal of Leisure Research, 39(2), 316-344.

Creswell, J. W. (2014). Research design: Qualitative, quantitative, and mixed methods approaches. (4th ed.). Thousand Oaks, CA: Sage.

Dotterer, A. M., McHale, S. M., \& Crouter, A. C. (2007). Implications of out-of-school activities for school engagement in African American adolescents. Journal of Youth and Adolescence, 36, 391-401. doi: 10.1007/s10964-006-9161-3

Edwards, M. B., Kanters, M. A., \& Bocarro, J. N. (2011). Opportunities for extracurricular physical activity in North Carolina middle schools. Journal of Physical Activity and Health, 8, 597-605.

Flintoff, A. (2008). Targeting Mr average: Participation, gender equity and school sport partnerships. Sport, Education and Society, 13(4), 393-411. doi: 10.1080/13573320802445017

Flintoff, A., Foster, R., \& Wystawnoha, S. (2011). Promoting and sustaining high quality physical education and school sport through 
school sport partnerships. European Physical Education Review, 17(3), 341-351. doi: 10.1177/1356336X11416731

Fredricks, J. A., \& Eccles, J. S. (2006). Is extracurricular participation associated with beneficial outcomes? Concurrent and longitudinal relations. Developmental Psychology, 42(4), 698-713. doi: 10.1037/0012-1649.42.4.698

Japan Senior High School Teachers Union. (2008). Final report of actual condition survey for issues of school-based extracurricular sports activity in 2006.

Kanagawa Prefectural Center of Physical Education. (2007). As regards a way of future system of registration and mediation of coach. Retrieved from www.pref.kanagawa.jp/uploaded/attachment/2426.pdf (January 22, 2015)

Lipscomb, S. (2007). Secondary school extracurricular involvement and academic achievement: A fixed effects approach. Economics of Education Review, 26, 463-472. doi: 10.1016/j.econedurev.2006.02.006

Macdonald, D. (2011). Like a fish in water: Physical education policy and practice in the era of neoliberal globalization. Quest, 63, 36-45.

Ministry of Education, Culture, Sports, Science and Technology in Japan. (1997). Report of investigative research for way of schoolbased extracurricular sports activity. Retrieved from http://www.mext.go.jp/b_menu/shingi/chousa/sports/001/toushin/971201.htm (January 22, 2015)

Ministry of Education, Culture, Sports, Science and Technology in Japan. (2012). National survey result of physical and athletic capacity. Retrieved from http://www.mext.go.jp/component/b_menu/otherl_icsFiles/afieldfile/2012/10/09/1326591_07.pdf (January 22, 2015)

Ministry of Education, Culture, Sports, Science and Technology in Japan. (2013). Report of investigative research for way of schoolbased extracurricular sports activity: Aim for school-based extracurricular sports activity that every student can bright. Retrieved from http://www.mext.go.jp/a_menu/sports/jyujitsu/_icsFiles/afieldfile/2013/05/27/1335529_1.pdf (January 22, 2015)

Sasakawa Sports Foundation. (2014). Sports white paper: Mission and potential of sports. Tokyo, Japan: Sasakawa Sports Foundation.

Schaefer, D. R., Simpkins, S. D., Vest, A. E., \& Price, C. D. (2011). The contribution of extracurricular activities to adolescent friendships: New insights through social network. Developmental Psychology, 47(4), 1141-1152. doi: 10.1037/a0024091

Shernoff, D. J., \& Vandell, D. L. (2007). Engagement in after-school program activities: Quality of experience from the perspective of participants. Journal of Youth and Adolescence, 36, 891-903. doi: 10.1007/s10964-007-9183-5

Shioya, K. (2002). External coaches and volunteers in school-based extracurricular sports activities. Journal of Health, Physical Education and Recreation, 52(4), 285-289.

Sport Council Wales. (2009). Young people's participation in sport. Sportsupdate, 62, 11-16.

Tokyo Metropolitan Board of Education. (2008). Companion of coaching school-based extracurricular activity for external coach. Retrieved from http://www.kyoiku.metro.tokyo.jp/press/bukatsu_tebiki.pdf (January 22, 2015)

Whiteley, R. F., \& Richard, G. (2012). Timetabling and extracurricular activities: A study of teachers' attitudes towards preparation time. Management in Education, 26(1), 6-12. doi: 10.1177/0892020611426894

Wiersma, L. D., \& Sherman, C. P. (2005). Volunteer youth sport coaches' perspectives of coaching education/certification and parental codes of conduct. Research Quarterly for Exercise and Sport, 76(3), 324-338. doi: 10.1080/02701367.2005.10599303

Williams, J. B., Hay, J. P., \& Macdonald, D. (2011). The outsourcing of health, sport and physical educational work: A state of play. Physical Education and Sport Pedagogy, 16(4), 399-415. doi: 10.1080/17408989.2011.582492 
